\title{
TWO METHODS OF CONSTRUCTING CONTRACTIONS AND REVISIONS OF KNOWLEDGE SYSTEMS
}

\section{INTRODUCTION}

The theory of nonmonotonic reasoning and the theory of belief revision share a very important subject. Both theories help to understand how it is possible rationally to pass from one knowledge system into another knowledge system that is in contradiction with the former one. In nonmonotonic reasoning this transition is accomplished by distinguishing between axioms ("explicit beliefs") and theorems ("implicit beliefs") and giving up the doctrine that more axioms must always yield more theorems. Thus my old (implicit) belief that Tweety can fly may well turn into disbelief after getting the information (acquiring the explicit belief) that Tweety is a penguin. A considerable limitation of this approach is that the new axiom must be (monotonically) consistent with the previous axioms, or else we get an inconsistent knowledge base.

The theory of belief revision on which I shall focus my attention is not subject to this restriction. In fact, it does not at all distinguish between axioms and theorems, or between beliefs and their reasons. The knowledge systems it takes into consideration are whole theories, where a theory, or knowledge set, is understood as a set of sentences closed under some appropriate logic $\mathrm{Cn}$. We shall assume that this logic includes classical propositional logic, that it is compact (i.e., if $A \in \mathrm{Cn}(M)$ then $A \in \operatorname{Cn}\left(M_{0}\right)$ for some finite $\left.M_{0} \subseteq M\right)$ and that it satisfies disjunction of premises (i.e., if $C \in \operatorname{Cn}(M \cup\{A\})$ and $C \epsilon$ $\operatorname{Cn}(M \cup\{B\})$, then $C \in \operatorname{Cn}(M \cup\{A \vee B\}))$. As a consequence of this, we have the deduction theorem for our logic (if $B \in \operatorname{Cn}(M \cup\{A\}$ ) then $A \rightarrow B \in \mathrm{Cn}(M))$. Finally we shall presuppose that the logic is monotonic (if $A \in \operatorname{Cn}(M)$ then $A \in \operatorname{Cn}(M \cup\{B\})$ ). Our language is to include the usual propositional operators $\neg, \&, \vee, \rightarrow$, and $\leftrightarrow$, and the propositional constants $T$ ("Truth") and $\perp$ ("Falsity"). The set of all sentences of the language is denoted by $L$, the set of all 
logical theorems $\{A: A \in \mathrm{Cn}(\varnothing)\}$ is denoted by LT. $M \subseteq \mathrm{L}$ is called consistent if $\perp \notin \mathrm{Cn}(M)$. We frequently write $M \vdash A$ for $A \in \mathrm{Cn}(M)$. Throughout this paper, variables $A, B, C$ etc. range over arbitrary sentences, and variables $M, N, M^{\prime}, N^{\prime}, M^{\prime \prime}$ etc. range over arbitrary sets of sentences.

How can such a holistic theory of belief revision handle inconsistent theories? I believe that Tweety can fly. What should I do if I come to know that Tweety in fact does not fly? As a first answer, I may be content with the advice that I first have to withdraw my belief that Tweety can fly, then add the belief that Tweety cannot and finally take the deductive closurc. (This advice has become known as the Levi identity.) A moment's reflection shows that the real problems are not solved by this move. How are contractions to be accomplished? Peter Gärdenfors founded and developed, partly in collaboration with David Makinson and Carlos Alchourrón, the approach I am going to sketch now. At the end of the 1970s he started by specifying a set of "rationality postulates" or constraints that must be satisfied by operations on a knowledge set in order to model the intuitive process of knowledge contraction. Let $K$ be a knowledge set and $\dot{-}_{K}$ be a function from $L$ to $2^{\mathrm{L}}$. We write $K \dot{-A}$ for $\dot{ }_{K}(A)$. Then $\dot{ }_{K}$ is a contraction function over $K$ if and only if it satisfies the following postulates:

$$
\begin{aligned}
& K \leftarrow A \text { is a knowledge set } \\
& K \dot{-} A \subseteq K \\
& \text { (closure) } \\
& \text { (inclusion) } \\
& \text { if } A \notin K \text { then } K \dot{-A}=K \\
& \text { (vacuity) } \\
& \text { if } \forall A \text { then } A \notin K-A \\
& \text { (success) } \\
& K \subseteq \operatorname{Cn}((K=A) \cup\{A\}) \\
& \text { (recovery) } \\
& \text { if } \vdash A \leftrightarrow B \text { then } K \dot{-A}=K \dot{-} B \\
& K \dot{-A} \cap K \dot{-} B \subseteq K \dot{-A} \& B \\
& \text { (preservation) } \\
& \text { if } A \notin K-A \& \mathrm{~B} \text { then } \\
& K-A \& B \subseteq K-A \\
& \text { (conjunction 1) } \\
& \text { (conjunction 2) }
\end{aligned}
$$

For the motivation of these constraints for contractions see [4], Chapter 3, [5], and [7]. (C1)-(C8) make sure, among other things, that contractions do not incur unnecessary loss of information.' As we will 
almost exclusively be concerned with the formal aspects of the dynamics of knowledge systems in the following, some familiarity with the literature cited is useful for the reader.

Even if we dispose of a reasonable set of postulates for contractions, this will not help us in actually performing any contraction or revision of our current knowledge system. What we need is a method of constructing a new state of knowledge out of the earlier onc. We require some structure here to guide us which in a way corresponds to the information contained in the distinction between explicit and implicit beliefs in nonmonotonic reasoning, or in the storage of the justifications for our beliefs in reason maintenance systems. In this paper I shall briefly review two different methods of knowledge contraction that were suggested in the course of the Gärdenforsian research program. Then a tight connection between these methods will be established showing that they even can be viewed as equivalent. I close with some remarks on simple and iterated revisions.

\section{TWO ORDERINGS FOR CONTRACTION CONSTRUCTION}

The first method I want to discuss is investigated in Alchourrón, Gärdenfors and Makinson [2] and may be called the method of relational partial meet contractions. When trying to exclude $A$ from $K$ while minimizing the loss of information, it is tempting to take a maximal subset of $K$ that does not imply $A$. Let $M(K, A)$ be the set of all such subsets. Alchourrón and Makinson [1] showed, however, that the choice of an element of $M(K, A)$ will in general leave far too many sentences untouched. Conversely, they proved that the full meet $\cap M(K, A)$ cancels far too many sentences from $K$. A natural idea is to consider the partial meet of some preferred clements of $M(K, A)$. Provided that we have a preference relation on $M(K)$, where $M(K)=\bigcup\left\{M(K, A): \|^{4} A\right\},{ }^{2}$ we can obtain reasonable contractions:

DEFINITION 1. If $\leqslant$ is a preference relation on $M(K)$ then the associated contraction function $-=C(\leqslant)$ over $K$ is given by

$$
K-A=\left\{\begin{array}{cc}
\cap\left\{M \in M(K, A): M^{\prime} \leqslant M\right. & \\
\text { for all } \left.M^{\prime} \in M(K, A)\right\}, & \text { if } \forall A, \\
K, & \text { if } \vdash A .
\end{array}\right.
$$


If $\dot{-}$ is a contraction function over $K$ and there is a (transitive) preference relation $\leqslant$ on $M(K)$ such that $\dot{-}=C(\leqslant)$, then - is called a (transitively) relational partial meet contraction function $((T) R P M C F)$.

Note that $M(K, A)=\varnothing$ if and only if $A \in \mathrm{LT}$. Now, what properties should we require for $\leqslant$ ? Alchourrón, Gärdenfors and Makinson [2] make use of only two postulates:

$$
\begin{aligned}
& \text { If } M(K, A) \neq \varnothing \text { then there is an } M \in M(K, A) \text { such } \\
& \text { that } M^{\prime} \leqslant M \text { for all } M^{\prime} \in M(K, A) \quad \text { (existence) } \\
& \text { if } M \leqslant M^{\prime} \text { and } M^{\prime} \leqslant M^{\prime \prime} \text { then } M \leqslant M^{\prime \prime} \\
& \text { (transitivity) }
\end{aligned}
$$

Only relations $\leqslant$ satisfying (P0) are intended to quality as preference relations ( $P$-relations). ${ }^{3}$ The authors show that $C(\leqslant)$ satisfies $(\mathrm{C} 1)$ $(\mathrm{C} 8)$ if $\leqslant$ satisfies $(\mathrm{P} 0)$ and $(\mathrm{P} 1)$. Conversely, they prove a representation theorem: Any contraction function - over $K$ satisfying $(\mathrm{Cl})-$ (C8) is a TRPMCF. In the proof, a preference relation $\leqslant=P^{*}(\dot{-})$ is constructed from a given contraction function - over $K$, and it is shown that $C\left(P^{*}(\dot{-})\right)=\dot{-}$. We put off the discussion of $P^{*}(\dot{-})$ until the end of Section 4.

A second method of constructing contractions, the method of epistemic entrenchment contractions, is found for the first time in Gärdenfors [4], Section 4.8, and presented more elegantly in Gärdenfors and Makinson [5]. Some relation over the sentences in a knowledge set is supposed to exist that reflects how decply entrenched these sentences are in our current state of knowledge. Provided that we have a relation of epistemic entrenchment on $L$, then, Gärdenfors and Makinson recommend that it should guide us in performing knowledge contractions as follows:

DEFINITION 2. If $\leq$ is a relation of epistemic entrenchment on $L$ then the associated contraction function $-=C(\leq)$ over $K$ is given by

$$
K \cdot A= \begin{cases}K \cap\{B: A<A \vee B\}, & \text { if } \forall A, \\ K, & \text { if } \vdash A .\end{cases}
$$


If - is a contraction function over $K$ and there is a relation of epistemic entrenchment $\leq$ on $\mathrm{L}$ such that $\dot{-}=C(\leq)$, then - is called an epistemic entrenchment contraction function (EECF).

There should be no danger of confusion of this ' $C$ ' with the ' $C$ ' of Definition 1 if the different arguments are carefully kept apart. $A<A \vee B$ of course stands for $A \leq A \vee B$ and not $A \vee B \leq A$. We ought to say what features make a relation on $\mathrm{L}$ count as a relation of epistemic entrenchment (EE-relation). In [5] we find the following collection of postulates:

(E1) if $A \leq B$ and $B \leq C$ then $A \leq C$ (transitivity)

$$
\begin{array}{ll}
A \leq B \text { or } B \leq A & \text { (connectivity) } \\
\text { if } A+B \text { then } A \leq B & \text { (dominance) } \\
A \leq A \& B \text { or } B \leq A \& B & \text { (conjunctiveness) } \\
\text { if } K \neq \mathrm{L}, \text { then } A \leq B \text { for all } B \text { iff } A \notin K \\
\text { (minimality) } \\
\text { if } B \leq A \text { for all } B \text { then } A \in \mathrm{LT} & \text { (maximality) }
\end{array}
$$

Note that (E2), following from (E1), (E3) and (E4), is redundant. Nevertheless it is expedient to have it as a principle of its own. For example, (E2) immediately entails that $\leq$ is reflexive. Since $K$ appears in (ES), we call $\leq$ an EE-relation with respect to $K$. For the intuitive motivation of these postulates, sce [3], [4], [5].

The content of Definition 2 is not easy to grasp. But it receives an excellent justification from its interplay with the (re-)construction of EE-orderings out of the contraction behaviour.

DEFINITION 3. If - is a contraction function over $K$ then the associated relation of epistemic entrenchment $\leq=E(\dot{-})$ on $\mathrm{L}$ is given by

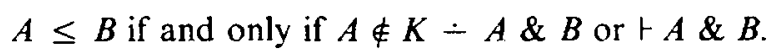

This definition is very plausible. If you give up $A \& B$ by retracting $A$ then $A$ could not have been more firmly entrenched than $B$. 


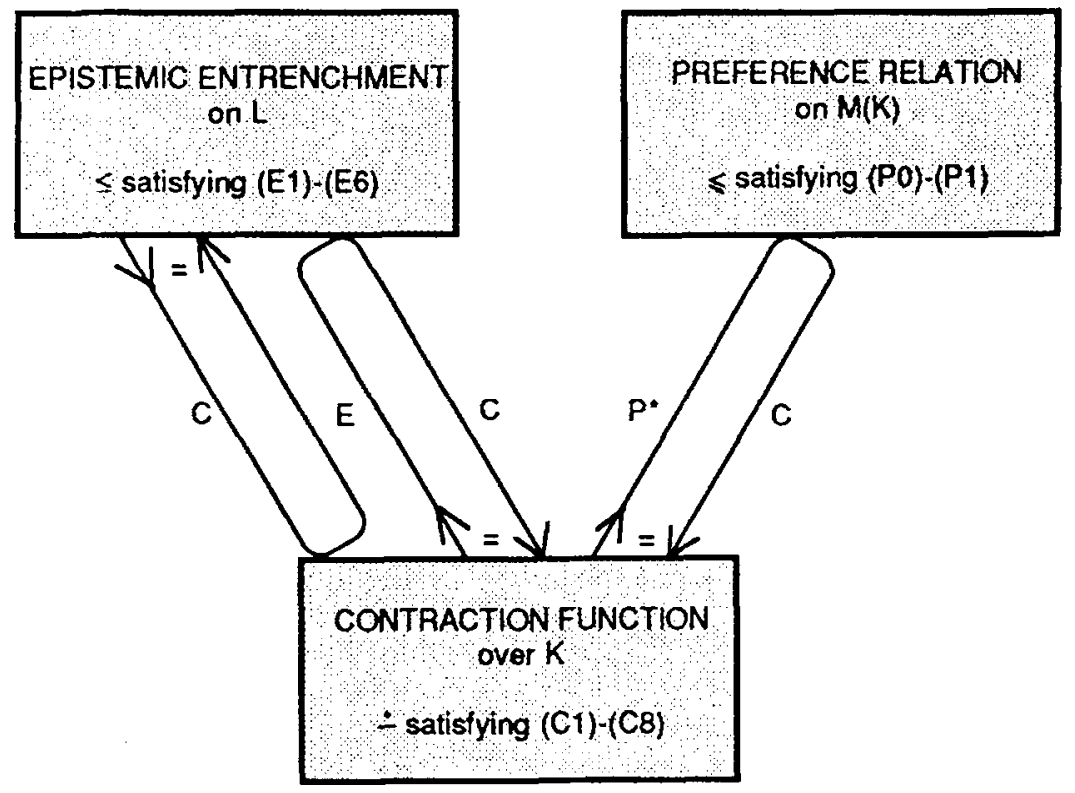

Scheme 1

In Gärdenfors and Makinson [5] we find the following important results: If $\leq$ satisfies (E1)-(E6) then $C(\leq)$ satisfies $(\mathrm{C} 1)-(\mathrm{C} 8)$; if - satisfics (C1)-(C8) then $E(-)$ satisfies (E1)-(E6); and finally, $E(C(\leq))=\leq$ for any $\leq$ satisfying (E1)-(E6), and $C(E(\dot{-}))=-$ for any - satisfying $(\mathrm{Cl})-(\mathrm{C} 8)$. Thus we get a kind of representation theorem similar to the first one: Any contraction function - over $K$ satisfying $(\mathrm{Cl})-(\mathrm{C} 8)$ is an EECF.

To sum up, we find ourselves faced with Scheme 1.

In my view, it is more natural to have a relation $\leq$ on $\mathrm{L}$ than a relation $\leqslant$ on $2^{\mathrm{L}}$. On the other hand, the rationale behind (T)RPMCFs is easier to understand than that behind EECFs. So both kinds of contraction constructions have their intuitive merits. It seems very desirable to know something about the relationship 
between (T)RPMCFs and EECFs. This is what we shall be concerned with in the main part of this paper.

\section{CONNECTING THE TWO ORDERINGS: GÄRDENFORS' FIRST ATTEMPT}

The first attempt to connect P-relations and EE-relations is due to Gärdenfors [3]. In this paper, however, Gärdenfors uses a different set of postulates for epistemic entrenchment. It does not include the limiting case conditions (E5) and (E6), and instead of (E4) it includes an apparent dual, viz.,

$$
A \vee B \leq A \text { or } A \vee B \leq B \text { (disjunctiveness). }
$$

There is also another postulate for the preference relation $\leqslant$ (which has already been discussed in [2]):

$$
M \leqslant M^{\prime} \text { or } M^{\prime} \leqslant M \quad \text { (connectivity). }
$$

Gärdenfors [3], pp. 359-360, then builds a two-way bridge between $\leq$ and $\leqslant$. For any given P-relation $\leqslant$, an EE-relation $\leq=E(\leqslant)$ is associated with $\leqslant$ by defining $A \leq B$ if and only if for all $M$ in $M(K)$ such that $A \in M$ there is an $M^{\prime}$ in $M(K)$ such that $B \in M^{\prime}$ and $M \leqslant M^{\prime}$. Conversely, for any given EE-relation $\leq$, a P-relation $\leqslant=P(\leq)$ is associated with $\leq$ by defining $M \leqslant M^{\prime}$ if and only if there is an $A \in M$ such that for all $B \in M^{\prime}$ it holds that $A \leq B$. Notice that these definitions look at the sentences that are retained in some elements of $M(K)$. Notice also that the second definition attaches most importance to the epistemically least entrenched sentences in $M$ and $M^{\prime}$, a fact which might raise some intuitive suspicion.

The formal results of Gärdenfors [3] are impressive. He shows that if $\leqslant$ satisfies $(\mathrm{P} 1)$ and $(\mathrm{P} 2)$, then $E(\leqslant)$ satisfies (E1)-(E3) and (E4*). If $\leq$ satisfies (E1)-(E3) and $\left(\mathrm{E} 4^{*}\right)$, then $P(\leq)$ satisfies $(\mathrm{P} 1)-(\mathrm{P} 2)$, provided that $K$ is a finite knowledge set, in the sense that $C$ partitions it into finitely many equivalence classes. ${ }^{4}$ In the finite case, Gärdenfors shows that $P(E(\leqslant))$ and $\leqslant$ are identical relations on $M(K)$, provided that $\leqslant$ satisfies $(\mathrm{P} 1)$ and $(\mathrm{P} 2)$, and that $E(P(\leq))$ and $\leq$ are identical relations on $K$, provided that $\leq$ satisfies $(\mathrm{E} 1)-(\mathrm{E} 3)$ and $\left(\mathrm{E} 4^{*}\right)$. 
Unfortunately these results are somewhat depreciated by the observation that $\left(\mathrm{E} 4^{*}\right)$, when taken together with (E1)-(E3), has some undesirable consequences. To prove this, I shall call $A$ an ordinary sentence if $A<\mathrm{T}$; if $\mathrm{T} \leq A$, then $A$ is called an extraordinary sentence. (Note that $A \leq \mathrm{T}$ by (E3).)

LEMMA 1. Let $\leq$ be a relation satisfying $(E I)-(E 3)$ and $\left(E 4^{*}\right)$ and let $A$ and $B$ be ordinary sentences. Then the biconditional $A \leftrightarrow B$ is extraordinary.

Proof. Let $\leq, A$ and $B$ be as indicated. We start by noting that $\vdash(A \vee B) \vee(A \leftrightarrow B)$. By $\left(\mathrm{E} 4^{*}\right)$ we have $(A \vee B) \vee(A \leftrightarrow B) \leq$ $A \vee B$ or $(A \vee B) \vee(A \leftrightarrow B) \leq A \leftrightarrow B$. The first condition cannot hold, however, since by hypothesis $A<\mathrm{T} \leq(A \vee B) \vee$ $(A \leftrightarrow B)$ and $B<T \leq(A \vee B) \vee(A \leftrightarrow B)$, whence (E1) would license to infer $A<A \vee B$ and $B<A \vee B$, in contradiction with (E4*). Thus the second condition must hold, which means that $A \leftrightarrow B$ is extraordinary.

$(E 4 *)$ is not really "dual" to (E4), but incompatible with it, on pain of triviality:

COROLLARY. Let $\leq$ be an EE-relation satisfying (EI)-(E4) and $\left(E 4^{*}\right)$, and let $A$ and $B$ be ordinary sentences. Then $A \leq B$ and $B \leq A$.

Proof. By Lemma 1 and (E3) we know that $A \vee \neg B$ is extraordinary. Hence $A<A \vee \neg B$. But by (E4) we have $A \vee B \leq$ $(A \vee B) \&(A \vee \neg B) \leq A$ or $A \vee \neg B \leq(A \vee B) \&$ $(A \vee \neg B) \leq A$. Thus $A \vee B \leq A$. But by an entirely analogous argument, also $A \vee B \leq B$. On the other hand, (E3) gives us $A \leq A \vee B$ and $B \leq A \vee B$. Hence, by (E1), $A \leq B$ and $B \leq A$.

Lemma 1 indeed shows that (E4*) is fatal. There are good reasons to represent all beliefs concerning particular matters of fact by ordinary sentences (compare (E6) above). There are also good reasons to keep extraordinary sentences in contractions as long as possible. But combining this and Lemma 1 entails that giving up any of our factual beliefs must result in giving up all of our factual beliefs, which is absurd. 
It seems to me, then, that we have to reject $\left(E 4^{*}\right)$. Yet we do not have to renounce results of the Gärdenfors [3] style. In the next section, I shall suggest different connections between E-relations and P-relations and then mimic Gärdenfors' theorems (wherc the finiteness assumption is dispensed with in all but one case). Furthermore, I show that EECFs and TRPMCFs are equivalent if the new bridge is crossed in either of the two possible ways.

\section{THF. RFAI, CONNECTION}

We begin by extending $\leqslant$ to an ordering of arbitrary sets of sentences, i.e., to a relation on $2^{\mathrm{L}}$. Henceforth we will call $\leqslant$ a preference relation, or $P$-relation, with respect to a knowledge set $K$ if and only if $\leqslant$ satisfies the following collection of postulates:

$$
\begin{array}{ll}
\text { If } M(K, A) \neq \varnothing \text { then there is an } M \in M(K, A) \text { such } \\
\text { that } N \leqslant M \text { for all } N \in M(K, A) & \text { (existence) } \\
\text { if } M \leqslant N \text { and } N \leqslant M^{\prime} \text { then } M \leqslant M^{\prime} & \text { (transitivity) } \\
M \leqslant N \text { or } N \leqslant M & \text { (connectivity) } \\
\text { if } M \subseteq N \text { then } M \leqslant N & \text { (dominance) } \\
M \leqslant M \cap N \text { or } N \leqslant M \cap N & \text { (intersection) } \\
M \leqslant N \text { for all } N \text { iff LT } \nsubseteq M & \text { (minimality) } \\
\text { if } K \neq \mathrm{L}, \text { then } N \leqslant M \text { for all consistent } & N \\
\text { iff } K \subseteq M & \text { (maximality) }
\end{array}
$$

As in the case of EE-relations, connectivity is redundant, but we shall keep it as a postulate of its own in order to sce its power. In contrast to EE-relations (see (E3)), P-relations seem to allow some kind of informational-content reading if we look only at (P3). However, (P4) shows that onc must be cautious with interpretations. I do not want to discuss the intuitive content of (P0)-(P6) here. In any case, these postulates will turn out to be suitable, when we follow a new road from $\leqslant$ to $\leq$ and vice versa.

DEFINTION 4. If $\leqslant$ is a P-relation on $2^{\text {l- }}$ (with respect to a knowledge set $K$ ) then the associated EE-relation $\leq=E(\leqslant)$ on $\mathrm{L}$ (with 
respect to $K$ ) is given by

$$
\begin{array}{cl}
A \leq B & \text { if and only if for all } M \text { in } M(K) \text { such that } \\
& B \notin M \text { there is an } M^{\prime} \text { in } M(K) \text { such that } \\
& A \notin M^{\prime} \text { and } M \leqslant M^{\prime} .
\end{array}
$$

We recall from [2], Lemma 2.4 , that for any $A \in K$ and $M \in M(K)$, $A \notin M$ is equivalent to $M \in M(K, A)$.

DEFINITION 5. If $\leq$ is an EE-relation on $L$ (with respect to a knowledge set $K$ ) then the associated P-relation $\leqslant=P(\leq)$ on $2^{\mathrm{L}}$ (with respect to $K$ ) is given by

$$
\begin{aligned}
& M \leqslant N \text { if and only if for all } A \notin N \text { there is a } B \notin M \\
& \text { such that } A \leq B .
\end{aligned}
$$

Notice that these definitions put special attention to the sentences that are missing in some set of sentences, and that Definition 5 attaches most importance to the epistemically most entrenched sentences missing. I believe that these features are intuitively more appealing than those of the Gärdenforsian definitions mentioned in Section 3.

Definitions 4 and 5 are formulated in close analogy to the Gärdenfors definitions. We are now going to state some useful equivalent formulations in two technical lemmas.

LEMMA 2. Let $\leqslant$ be a P-relation on $2^{l}$ with respect to a knowledge set of $K$. Then the following three conditions are equivalent:

(i) For all $M$ in $M(K)$ such that $B \notin M$ there is an $M^{\prime}$ in $M(K)$ such that $A \notin M^{\prime}$ and $M \leqslant M^{\prime}$;

(ii) for all knowledge sets $M$ such that $B \notin M$ there is a knowledge set $N$ such that $A \notin N$ and $M \leqslant N$;

(iii) for all $M \in 2^{L}$ such that $M \forall B$ there is an $N \in 2^{L}$ such that $N \forall A$ and $M \leqslant N$.

Proof. From (i) to (ii): Let $M$ be a knowledge such that $B \notin M$. By (P4), (P6) and (P1), we get $M \leqslant M \cap K$. Since $M \cap K$ is again a knowledge set and $B \notin M \cap K$, there is an $M^{\prime}$ in $M(K, B)$ such that $M \cap K \subseteq M^{\prime}$, the latter giving us $M \cap K \leqslant M^{\prime}$, by (P3). By application of (i) we get that there is an $M^{\prime \prime}$ in $M(K)$ such that $A \notin M^{\prime \prime}$ 
and $M^{\prime} \leqslant M^{\prime \prime}$. Since elements of $M(K)$ are knowledge sets and since $M \leqslant M \cap K \leqslant M^{\prime} \leqslant M^{\prime \prime}$, we find that, by (P1), $M^{\prime \prime}$ is an $N$ we are looking for in (ii).

From (ii) to (iii): Let $M \in 2^{\mathrm{L}}$ be such that $M \nvdash B$. Let $M^{\prime}=$ $\mathrm{Cn}(M)$ is a knowledge set, $B \notin M^{\prime}$ and, by (P3), $M \leqslant M^{\prime}$. By application of (ii) we get that there is a knowledge set $N$ such that $A \notin N$ and $M^{\prime} \leqslant N$. Since knowledge sets are closed under $\mathrm{Cn}, N \nLeftarrow A$, and we find that, by (P1), $N$ is just such an $N$ as we are looking for in (iii).

From (iii) to (i): Let $M$ in $M(K)$ be such that $B \notin M$. Since elements of $M(K)$ arc knowledge sets, $M \forall B$. By application of (iii) we get that there is an $N \in 2^{\mathrm{L}}$ such that $N H A$ and $M \leqslant N$. Set $N^{\prime}=\mathrm{Cn}(N)$; then $A \notin N^{\prime}$ and, by (P3), $N \leqslant N^{\prime}$. By (P4), (P6) and (P1), we get $N^{\prime} \leqslant N^{\prime} \cap K$. Since $N^{\prime} \cap K$ is a knowledge set and $A \notin N^{\prime} \cap K$, there is an $M^{\prime}$ in $M(K, A)$ such that $N^{\prime} \cap K \subseteq M^{\prime}$, the latter giving us $N^{\prime} \cap K \leqslant M^{\prime}$, by (P3). By $M \leqslant N \leqslant N^{\prime} \leqslant N^{\prime} \cap$ $K \leqslant M^{\prime}$ and (P1), we find that $M^{\prime}$ is just such as $M^{\prime}$ as we are looking for in (i).

It will be convenient to have an additional technical concept in the following. If $\leq$ is an EE-relation on $\mathrm{L}$, then an EE-cut, or simply cut, is a set of sentences $S$ such that for any $A$ in $S$ all sentences $B$ with $A \leq B$ are also in $S .{ }^{5}$ We note some properties of cuts. It is easy to verify that the class of all cuts is closed under arbitrary unions and intersections, that cuts are nested (by (E2)), and that $S_{A}$, defined by $\{B: A<B\}$, and $S_{A}$, defined as $\{B: A \leq B\}$, are cuts by (E1)). The largest cut is $L=\{B: \perp \leq B\}$, then comes $K=\{B: \perp<B\}$ (if $K \neq \mathrm{L}$ ), then come subsets of $K$, the smallest but one cut is LT $=$ $\{B: T \leq B\}$ and the smallest cut is $\varnothing=\{B: T<B\}$ (by (E5), (E3) and (E6)). Any non-empty cut $S$ is a knowledge sct, provided that $\mathrm{Cn}$ is compact. To see this, assume that $S-A$. Then $B_{1} \& \ldots \& B_{n} \vdash A$ for some $B_{1}, \ldots, B_{n}$ in $S$, hence, by (E3), $B_{1} \& \ldots \& B_{n} \leq A$. Iterated application of (E4) gives us $B_{i} \leq B_{1} \& \ldots \& B_{n}$ for some $i \in\{1, \ldots, n\}$, hence, by (E1), $B_{i} \leq A$. But as $B_{i} \in S$ and $S$ is a cut, this entails that $A \in S$, and we are done.

We are now able to recast the defining condition of Definition 5. 
LEMMA 3. Let $\leq$ be an EE-relation on $L$. Then the following two conditions are equivalent:

(i) For all $A \notin N$ there is a $B \notin M$ such that $A \leq B$.

(ii) For all cuts $S$, if $S \subseteq M$ then $S \subseteq N$.

Proof. From (i) to (ii): (i) is equivalent to the condition that $S_{A}=$ $\{B: A \leq B\} \nsubseteq M$ for all $A \notin N$. By contraposition, if $S_{A} \subseteq M$ then $A \in N$. Let $S$ be a cut such that $S \subseteq M$. We have to show that $S \subseteq N$. But for any $A$ in $S, S_{A} \subseteq S \subseteq M$, hence $A \in N$, so $S \subseteq N$.

From (ii) to (i): (ii) entails that for all $A$, if $S_{A} \subseteq M$, then $S_{A} \subseteq N$. Hence, by contraposition and by the reflexivity of $\leq, S_{\mathrm{A}} \nsubseteq M$ for all $A \notin N$. But this just means that there is a $B$ such that $A \leq B$ and $B \notin M$, for all $A \notin N$, and we are done.

Notice that the proof of Lemma 3 makes use only of the transitivity and connectivity of $\leq$. In the following we shall frequently work with the nicer conditions (iii) of Lemma 2 and (ii) of Lemma 3 in lieu of the characterizations of $E(\leqslant)$ and $P(\leq)$ given in Definitions 4 and 5 . We are now going to explore the interrelation between EErelations and P-relations.

LEMMA 4. Let $\leqslant$ be a P-relation on $2^{L}$ (with respect to a knowledge set $K)$. Then $\leq=E(\leqslant)$ satisfies $(E I)-(E 6)$.

Proof. (E1) is easy and (E2) follows from (E1), (E3) and (E4). For (E3), suppose that $A \vdash B$ and $M \forall B$. In order to get $A \leq B$, we have to show that there is an $N$ such that $N \forall A$ and $M \leqslant N$. But $M \forall A$, and by the reflexivity of $\leqslant$ (which in turn follows from (P2)), $M \leqslant M$, so we can put $N=M$. - For (E4), suppose for reductio ad absurdum that neither $A \leq A \& B$ nor $B \leq A \& B$. This means that there is an $M$ such that $M \forall A \& B$ and for all $N$ such that $N \forall A$ it holds that not $M \leqslant N$, and there also is an $M^{\prime}$ such that $M^{\prime} \forall A \& B$ and for all $N^{\prime}$ such that $N^{\prime} \forall B$ it holds that not $M^{\prime} \leqslant N^{\prime}$. Since $\leqslant$ is reflexive, $M \vdash A$ and $M^{\prime} \vdash B$. Hence $M \forall B$ and $M^{\prime} \forall A$. Hence neither $M \leqslant M^{\prime}$ nor $M^{\prime} \leqslant M$, contradicting (P2). - For (E5), let $K \neq \mathrm{L}$. Assume that $A \leq B$ for all $B$. This means that for any $B$ and any $M$ such that $M \forall B$ there is an $N$ such that $N \forall A$ and $M \leqslant N$, which is equivalent to the statement that there is an $N$ such that $N \forall A$ and $M \leqslant N$ for all consistent $M$. By (P6) this in turn means that 
there is an $N$ such that $N \forall A$ and $K \subseteq N$, which reduces to $K \forall A$ or, as $K$ is a knowledge set, to $A \notin K$. - For (E6), assume that $B \leq A$ for all $B$. This means that for any $B$ and any $M$ such that $M \forall A$ there is an $N$ such that $N \forall B$ and $M \leqslant N$. But if we take $T$ as $B$, it is impossible that $N \dot{\forall} B$. Thus it is impossible that $M \forall A$ for any $M$, thus $1 A$.

Note that the proof of Lemma 4 does not use (P0), (P3), (P4) or (P5).

LEMMA 5. Let $\leq$ be an EE-relation on $L$ (with respect to a knowledge set $K)$. Then $\leqslant=P(\leq)$ satisfies $(P 0)-(P 6)$.

Proof. For (P0), assume that $M(K, A) \neq \varnothing$. Then $t^{\prime} A$. We have to show that there is an $M \in M(K, A)$ such that for all $M^{\prime} \in M(K, A)$, $M^{\prime} \leqslant M$, the latter meaning that every cut $S \subseteq M^{\prime}$ is a subset of $M$. We first note that the largest cut that can be a subset of any element of $M(K, A)$ is $S_{A}=\{B: A<B\}$, since every larger cut includes $A$ (by the reflexivity of $\leq$ ). Second, $S_{A} \neq \varnothing$. by (E5). A third observation is that the non-empty cut $S_{A}$ is a knowledge set which does not contain $A$, hence $S_{A} \forall A$. But now we see that $M(K, A)$ must contain a superset $M$ of $S_{A}$. Clearly for any $M^{\prime} \in M(K, A)$ and any cut $S$, if $S \subseteq M^{\prime}$ then $S \subseteq S_{A} \subseteq M$, and we are done. - (P1) is immediate, and (P2) follows from (P1), (P3) and (P4). - For (P3), assume that $M \subseteq N$. For $M \leqslant N$, we have to verify that every cut $S \subseteq M$ is a subset of $N$, which is trivial. - For (P4). suppose for reductio ad absurdum $M \notin M \cap N$ and $N \notin M \cap N$. This means that there is a cut $S$ such that $S \subseteq M$ and $S \nsubseteq M \cap N$ and a cut $S^{\prime}$ such that $S^{\prime} \subseteq N$ and $S^{\prime} \nsubseteq M \cap N$. From $S \subseteq M$ and $S^{\prime} \subseteq N$ we get $S \cap S^{\prime} \subseteq M \cap N$. But since cuts are nested, $S \cap S^{\prime}=S$ or $S \cap S^{\prime}=S^{\prime}$, and we get a contradiction. - For (P5), assume that $M \leqslant N$ for all $N$. This means that for any $N \in 2^{\mathrm{L}}$ and any cut $S$, if $S \subseteq M$ then $S \subseteq N$, or equivalently, that for any cut $S$, if $S \subseteq M$ then $S=\varnothing$. Since LT is the second smallest cut (which is due to (E6)), the latter is equivalent to $A \notin M$ for some $A$ such that $\vdash A$. For (P6), let $K \neq \mathrm{L}$. Assume that $N \leqslant M$ for every consistent $N$. This means that for every consistent $N$ and every cut $S$, if $S \subseteq N$ then $S \subseteq M$, or equivalently, since $K \neq \mathrm{L}$ is the second largest cut (which is duc to (E5)), $K \subseteq M$. 
Note that the proof of Lemma 5 requires all of the postulates (E1)(E6), which already enter into our knowledge of cuts.

THEOREM 1. Let $\leq$ be an EE-relation on $L$ (with respect to a knowledge set $K)$. Then $\leq^{*}=E(P(\leq))$ is identical with $\leq$.

Proof. We have to show that $A \leq^{*} B$ if and only if $A \leq B$.

From left to right: Let $A \leq^{*} B$, which means that for all $M$ such that $M \forall B$ there is an $N$ such that $N \forall A$ and for all cuts $S$, if $S \subseteq M$ then $S \subseteq N$. Suppose for reductio that $B<A$, i.c., that $A \in S_{B}$. Since $S_{B}$ is a knowledge set not containing $B, S_{B} \forall B$. Thus there must be an $N$ such that $N \forall A$ and $S_{B} \subseteq N$. But since $A \in S_{B}$, $N$ contains $A$, so $N+A$, and we have a contradiction.

From right to left: Let $B<^{*} A$, which means that there is an $M$ such that $M \forall B$ and for all $N$ such that $N \forall A$ there is a cut $S$ such that $S \subseteq M$ and $S \nsubseteq N$. Suppose for reductio that $A \leq B$, i.e., that $A \notin S_{B}$. Since $S_{B}$ is a knowledge set, $S_{B} \not A$. Thus there must be an $M$ such that $M \sharp B$ and a cut $S$ such that $S \subseteq M$ and $S \nsubseteq S_{B}$. From the latter condition it follows that there is a $C$ in $S$ such that $C \leq B$, and since $S$ is a cut, $B$ must be in $S$ too. But as $S \subseteq M$, then $B \in M$, so $M \vdash B$, and we have a contradiction.

THEOREM 2. Let $\leqslant$ be a P-relation on $2^{L}$ with respect to a finite knowledge set $K$. Then the restrictions of $\leqslant^{*}=P(E(\leqslant))$ and $\leqslant$ to the class of knowledge sets are identical.

Proof. Let $K$ be a finite knowledge set. We have to show that for any two knowledge sets $M$ and $N, M \leqslant *$ iff $M \leqslant N$. Let $N$ and $M$ always stand for knowledge sets in this proof. The cut notation is not useful here, so we apply the original condition of Definition 5.

From left to right: Let $M \leqslant^{*} N$, which means that for all $A \notin N$ there is a $B \notin M$ such that for all $M^{\prime}$ with $M^{\prime} \forall B$ there is an $N^{\prime}$ such that $N^{\prime} \forall A$ and $M^{\prime} \leqslant N^{\prime}$. As $M$ is a knowledge set, we can put $M^{\prime}=M$, getting that for all $A \notin N$ there is an $N^{\prime}$ such that $N^{\prime} \forall A$ and $M \leqslant N^{\prime}$. In particular, for all $A \in K-N$ there is an $N^{\prime}$ such that $N^{\prime} H A$ and $M \leqslant N^{\prime}$. Since $K$ is finite modulo $C n$, we will need only finitely many such sets $N^{\prime}$. Call them $N_{\mathrm{l}}^{\prime}, \ldots, N_{n}^{\prime}$. Since $N^{\prime}$ HA entails $A \notin N^{\prime}$, we have $\left(N_{1}^{\prime} \cap \ldots \cap N_{n}^{\prime}\right) \cap K \subseteq N \cap K$, hence $\left(N_{1}^{\prime} \cap \ldots \cap N_{n}^{\prime}\right) \cap K \leqslant N \cap K$. On the other hand, by $(\mathrm{n}-1)$-fold 
application of (P4), $N_{i}^{\prime} \leqslant N_{1}^{\prime} \cap \ldots \cap N_{n}^{\prime}$ for some $i \in\{1, \ldots, n\}$. Furthermore, (P4), (P6) and (P1) give us $N_{1}^{\prime} \cap \ldots \cap N_{n}^{\prime} \leqslant\left(N_{1}^{\prime} \cap \ldots \cap\right.$ $\left.N_{n}^{\prime}\right) \cap K$. Putting all this together, we finally get the chain $M \leqslant N_{i}^{\prime} \leqslant$ $N_{1}^{\prime} \cap \ldots \cap N_{n}^{\prime} \leqslant\left(N_{1}^{\prime} \cap \ldots \cap N_{n}^{\prime}\right) \cap K \leqslant N \cap K \leqslant N$, and, by (P1), we arrive at $M \leqslant N$.

From right to left: Let $N<^{*} M$, which means that there is an $A \notin N$ such that for all $B \notin M$ there is an $M^{\prime}$ with $M^{\prime} \forall B$ such that for all $N^{\prime}$ with $N^{\prime} H A, N^{\prime}<M^{\prime}$. As $N$ is a knowledge set, we can put $N^{\prime}=N$, getting that for all $B \notin M$ there is an $M^{\prime}$ such that $M^{\prime} \forall B$ and $N<M^{\prime}$. By a chain of reasoning entirely analogous to the from-left-to-right case, we get $N<M$.

The last four results reproduce those of Gärdenfors [3] in our new setting. The main differences are these: first, I use more complete sets of conditions for $\leq$ and $\leqslant$; second, I consider $\leq$ as a relation not only on $K$ but on $\mathrm{L}$ and $\leqslant$ as a relation not only on $M(K)$ but on $2^{\mathrm{L}}$; third, I need the finiteness assumption for $K$ only once where Gärdenfors uses it three times. The most interesting observations of this paper are, I believe, the following theorems for which there are no precedents in [3]. Roughly, they say that partial meet contractions based on $\leqslant$ and epistemic entrenchment contractions based on $\leq$ are equivalent, if $\leqslant$ and $\leq$ are related by either Definition 4 or Definition 5.

THEOREM 3. Let $\leqslant$ be a P-relation on $2^{L}$ (with respect to a knowledge set $K)$. Then $C(E(\leqslant))$ is identical with $C(\leqslant)$.

Proof. If we write - for $C(E(\leqslant))$ and $二^{*}$ for $C(\leqslant)$. we have to show that $K \dot{-A}=K \dot{-}^{*} A$ for any $A \in \mathrm{L}$. This is trivial in the

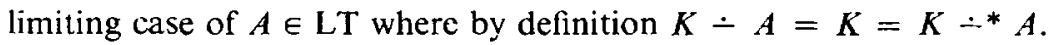
Now let $A \notin \mathrm{LT}$.

Condition (iii) of Lemma 2 is not useful here, so we go back to the original condition of Definition 4. For $K-A=K \dot{-}^{*} A$ then we have to show that

$K \cap\left\{B\right.$ : there is an $M^{\prime}$ in $M(K)$ such that $A \notin M^{\prime}$ and for all $M$ in $M(K)$ such that $A \vee B \notin M$ it holds that $\left.M<M^{\prime}\right\}=$

$\cap\left\{M \in M(K, A): M^{\prime} \leqslant M\right.$ for all $M^{\prime}$ in $\left.M(K, A)\right\}$. 
Consider the case where $A$ is not in $K$. Then $M(K, A)=\{K\}$, so the RHS of $\left(^{*}\right)$ is $K$. It remains to show that for all $B \in K$ there is an $M^{\prime}$ in $M(K)$ such that $A \notin M^{\prime}$ and for all $M$ in $M(K)$ such that $A \vee B \notin M$ it holds that $M<M^{\prime}$. Let $B \in K$ and choose $K$ as $M^{\prime}$. It suffices to show that for all $M$ in $M(K)$ such that $A \vee B \notin M$ we have $M<K$. But if $A \vee B \notin M$ then $K$ is not a subset of $M$, since $A \vee B \in K$. So, by (P6), $M<K$.

Now consider the principal case $A \in K$. We first note that for all $M$ in $M(K)$ and all $A$ and $B$ in $K, A \vee B \notin M$ iff $A \notin M$ and $B \notin M$. For suppose that $M \in M(K, C)$; then $A \vee B \in K-M$ iff $M \cup$ $\{A \vee B\} \vdash C$, i.c., $M \cup\{A\} \vdash C$ and $M \cup\{B\} \vdash C$, i.e., $A \in K-M$ and $B \in K-M$.

Using this we know that $B$ is in the LHS of $\left(^{*}\right)$ if and only if

$$
B \in K \text { and there is an } M^{\prime} \text { in } M(K) \text { such that } A \notin M^{\prime}
$$
and for all $M$ in $M(K)$ such that $A \notin M$ and $B \notin M$ it holds that $M<M^{\prime}$.

From $\left({ }^{* *}\right)$ we get by simple predicate calculus

(***) for all $M$ in $M(K)$ such that $A \notin M$ and $B \notin M$ there is an $M^{\prime}$ in $M(K)$ such that $A \notin M^{\prime}$ and $M<M^{\prime}$.

To see that conversely $\left({ }^{* * *}\right)$ already entails $\left({ }^{* *}\right)$, first suppose for reductio that $\left({ }^{* * *}\right)$ holds but $B \notin K$. Then $B \notin M$ for all $M$ in $M(K)$. By Lemma 2.4 of [2], $\left({ }^{* * *}\right)$ then states that for all $M \in M(K, A)$ there is an $M^{\prime} \in M(K, A)$ such that $M<M^{\prime}$. But this contradicts (P0). Thus $B \in K$. Second, suppose for reductio that $\left({ }^{* * *}\right)$ holds but also that for all $M^{\prime}$ in $M(K)$ such that $A \notin M^{\prime}$ there is an $M$ in $M(K)$ such that $A \notin M$ and $B \notin M$ and $M^{\prime} \leqslant M$. By (P1) and Lemma 2.4 of [2], then we get that for all $M^{\prime} \in M(K, A)$ there is an $M^{\prime \prime} \in M(K, A)$ such that $M^{\prime}<M^{\prime \prime}$. But this again contradicts (P0). Thus ( $\left.{ }^{* *}\right)$.

Now we know that $B$ is in the LHS of $\left({ }^{*}\right)$ iff $\left({ }^{* * *}\right)$ is satisfied. $\left({ }^{* * *}\right)$ is logically equivalent to $B \in \bigcap\left\{M \in M(K): A \notin M\right.$ and $M^{\prime} \leqslant M$ for all $M^{\prime} \in M(K)$ such that $\left.A \in M^{\prime}\right\}$. Using Lemma 2.4 of [2] again, we see that this may be expressed as $B \in \cap\left\{M \in M(K, A): M^{\prime} \leqslant M\right.$ for all $\left.M^{\prime} \in M(K, A)\right\}$, which is just the RHS of $\left(^{*}\right)$.

Using previously established results, we obtain the next central theorem as a corollary of Theorem 3. 


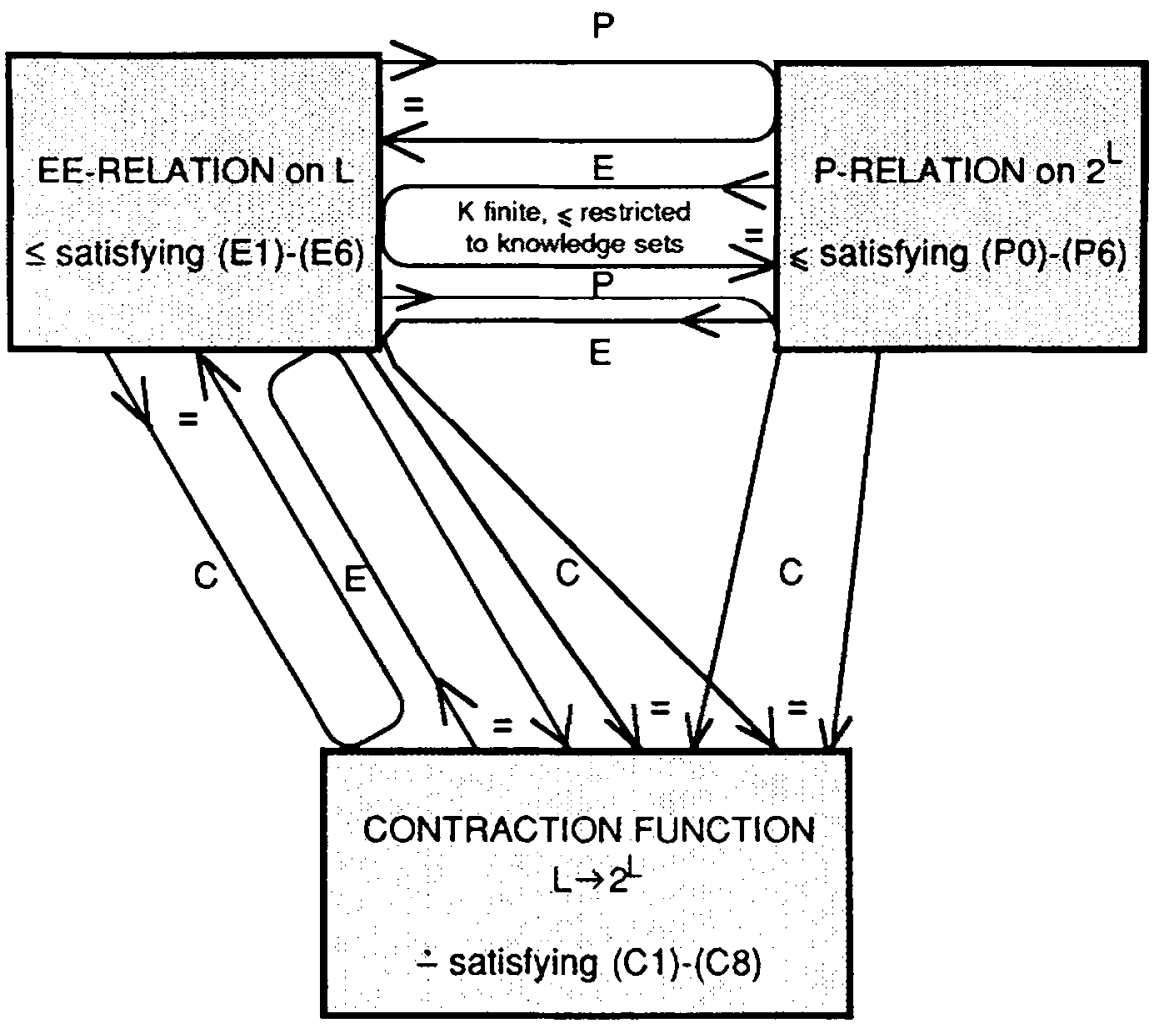

Scheme 2

THEOREM 4. Let $\leq$ be an EE-relation on $L$ (with respect to knowledge set $K)$. Then $C(P(\leq))$ is identical with $C(\leq)$.

Proof. From Lemma 5 we know that $P(\leq)$ is a P-relation with respect to $K$. Hence, by Theorem 3, $C(P(\leq))=C(E(P(\leq)))$.

Theorem 1 tells us that $E(P(\leq))=\leq$, hence $C(E(P(\leq)))=C(\leq)$. Putting this together, we get $C(P(\leq))=C(\leq)$.

Taking over the connection between EE-relations and (EE-)contraction functions from [5], we are now presented Scheme 2. 
The missing links between preference relations and contraction functions are supplied easily by a last connecting definition.

DEFINITION 6. If - is a contraction function over $K$ then the associated preference relation $\leqslant=P(\dot{-})$ on $2^{\mathrm{L}}$ is given by $P(E(\dot{-}))$.

$P(-)$ of course satisfies (P0)-(P6). We can establish a number of corrollaries in a way similar to the proof of Theorem 4.

COROLLARIES. Let - be a contraction function over a knowledge set $K$, $\leq$ an EE-relation and $\leqslant$ be a P-relation with respect to $K$. Then the following identities hold:

(ii) $\quad P(C(\leqslant))=\leqslant$ within the class of knowledge sets, provided that $K$ is finite.

(iii) $\quad P(C(\leq))=P(\leq)$.

(iv) $\quad E(C(\leqslant))=E(\leqslant)$.

(v) $\quad E(P(\dot{-}))=E(\dot{-})$.

A last interesting question is how Definition 6 relates to the construction of a preference relation $P^{*}(\dot{-})$ which can be found in Alchourrón, Gärdenfors and Makinson [2], p. 519. Let $N(K, A)$ be the set of all $M$ in $M(K)$ such that $K \dot{-A} \subseteq M$, and $N(K)$ the set of all $M$ in $M(K)$ such that $K \dot{-} \subseteq \subseteq M$ for some $A$. Note that $N(K, A) \subseteq M(K, A) \cup$ $\{K\}$ : Let $M \in N(K, A)$; then $K \therefore A \subseteq M$ and, without loss of generality, $M \in M(K, B)$; if $B \notin K$ then $M=K$ and we are done; if $B \in K$ then, by (C5), $A \rightarrow B \in K \dot{-A} \subseteq M$, hence, since $M \forall B$, $A \notin M$. So, by Lemma 2.4 of [2], $M \in M(K, A)$.

$P^{*}(\dot{-})$ is defined on $2^{K}$; if we abbreviate it by $\leqslant^{*}$ it can be introduced as follows: $M \leqslant \leqslant^{*} M^{\prime}$ if and only if $M \in M(K), M^{\prime} \in N(K)$ and for all $A$, if $M \in N(K, A)$ and $M^{\prime} \in M(K, A)$ then $M^{\prime} \in N(K, A)$.

Since both $C\left(P^{*}(\dot{-})\right)=\dot{-}$ (see [2]) and $C(P(\dot{-}))=\dot{-}$ (corollary (i) above), one may expect that $P^{*}(\dot{-})$ and $P(\dot{-})$ show some agreement. Obviously it is only interesting to compare the restrictions of 
$P^{*}(\dot{-})$ and $P(\dot{-})$ to $M(K)$. I could not prove, nor do I think it is true, that in general $P^{*}(\dot{-})$ and $P(\dot{-})$ are identical within the whole range of $M(K)$. However, we can get the following result:

THEOREM 5. Let - be a contraction function over a knowledge set $K$. If $\leqslant=P(\dot{-})$ and $\leqslant^{*}=P^{*}(\dot{-})$, then $M \leqslant M^{\prime}$ iff $M \leqslant{ }^{*} M^{\prime}$ for all $M$ in $N(K)-\{K\}$ and $M^{\prime}$ in $N(K)$.

Sketch of proof. Let $M \in N(K)-\{K\}$ and $M^{\prime} \in N(K)$. We have to show that

for all $A \notin M^{\prime}$ there is a $B \notin M$ such that $A \notin K \dot{A} \& B$

if and only if

$(* *)$

$$
\begin{aligned}
& \text { for all } C \text {, if } M \in N(K, C) \text { and } M^{\prime} \in M(K, C) \\
& \text { then } M^{\prime} \in N(K, C) .
\end{aligned}
$$

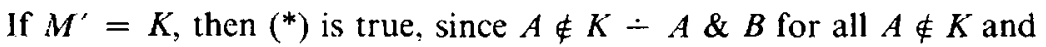
arbitrary $B$, and $\left({ }^{* *}\right)$ is true as well, since $K \in M(K, C)$ implies that $M(K, C)=\{K\}$. Let us now turn to the principal case $M^{\prime} \neq K$.

To sec that $\left({ }^{*}\right)$ implies $\left({ }^{* *}\right)$, suppose for reductio that $\left({ }^{*}\right)$ holds but not $\left({ }^{* *}\right)$, the latter meaning that $M \in N(K, C), M^{\prime} \in M(K, C)$ and $M^{\prime} \notin N(K, C)$ for some $C$, As $K \dot{-C} \nsubseteq M^{\prime}$, we can choose a $D$ such that $D \in K-C$ and $D \notin M^{\prime}$. Since $C, D \in K, C, D \notin M^{\prime}$ and $M^{\prime} \in M(K)$, we get, by an argument already used in the proof of Theorem 3,C $C \vee D \notin M^{\prime}$. By $\left(^{*}\right)$, there is a $B \notin M$ such that $C \vee D \notin$ $K-(C \vee D) \& B$. Next we show that $B \notin K-B \& C$ : by (C4), $B \notin K-B \& C$ or $C \notin K \div B \& C$; in the first case we are done and in the second case (C8) gives us $K-B \& C \subseteq K-C$, and since $K-C \subseteq M$ and $B \notin M$, we get $B \notin K-B \& C$. From $C \vee D \notin$ $K-(C \vee D) \& B$ and $B \notin K \sqcup B \& C$, we conclude with the help of a transitivity argument taken from [5], p. 94, that $C \vee D \notin K-$

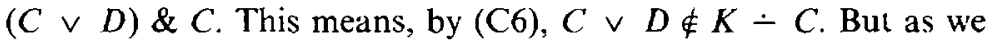
assumed $D \in K-C$, we have a contradiction with (Cl).

Conversely, to sec that $\left(^{* *}\right)$ implies $\left({ }^{*}\right)$, suppose for reductio that $\left.{ }^{* *}\right)$ holds, but not $\left({ }^{*}\right)$. The latter means that there is an $A \notin M^{\prime}$ such that $A \in K-A \& B$ for all $B \notin M$. Choose such an $A$. Without loss of generality, let $M \in N(K)-\{K\}$ be in $N(K, E)$. Hence $M \in M(K, E)$, so $E \notin M$, hence $A \in K-A \& E$. By (C1) and (C4), then, $E \notin K-$ $A \& E$, hence, by (C8), $K \doteqdot A \& E \subseteq K \div E$. Since $K-E \subseteq M$, 
also $K \dot{-A} \& E \subseteq M$. But as $A \notin M^{\prime}$, also $A \& E \notin M^{\prime}$, hence by Lemma 2.4 of [2] $M^{\prime} \in M(K, A \& E)$. Thus $\left(^{* *}\right)$ gives us $K-A \&$ $E \subseteq M^{\prime}$. But $A \in K \sqcup A \& E$ and $A \notin M^{\prime}$, so $K \sqcup A \& E \nsubseteq M^{\prime}$, and we have a contradiction.

For finite knowledge sets the agreement between $P^{*}(\dot{-})$ and $P(\dot{-})$ is almost perfect:

COROLLARY. Let - he a contraction function over a finite knowledge set $K$. If $\leqslant=P(\dot{-})$ and $\leqslant^{*}=P^{*}(\dot{-})$ then $M \leqslant M^{\prime}$ iff $M \leqslant{ }^{*} M^{\prime}$ for all $M$ in $M(K)-\{K\}$ and $M^{\prime}$ in $M(K)$.

Proof. In view of Theorem 5 it suffices to show that $M(K) \subseteq N(K)$ for finite $K$. Let $M \in M(K)$. We have to show that $K \dot{-} \subseteq \subseteq M$ for some $A$. Since $K$ is finite, $K-M$, too, is partitioned into finitely many equivalence classes by $\mathrm{Cn}$. Let $A$ be the disjunction $A_{1} \vee \ldots \vee A_{n}$ of representatives of these equivalence classes. We show that in fact $K \dot{-A \subseteq M}$. Let $B \in K-A$. By (C4) and (C1), $B \forall A$. So, by construction of $A, B \notin K-M$. But as $B \in K$ by (C2), we get $B \in M$. (It is possible to show that even $K \dot{-}=M$.)

\section{REMARKS ON SIMPLE REVISIONS}

At the end of Section 2 we noted that it is easier to conceive EErelations on $\mathrm{L}$ then $\mathrm{P}$-relations on $\mathbf{2}^{\mathrm{L}}$, but that EE-contraction functions are not very perspicuous. In many contexts, however, one is interested in contraction functions only as a means of obtaining revisions. We base the following considerations on the advice of the Levi identity which tells us that a revision function $\dot{+}=R(\dot{-})$ over a knowledge set $K$ is to be constructed from a contraction function over $K$ by putting $K+A=\mathrm{Cn}((\mathrm{K}-\neg A) \cup\{A\})$ for all sentences $A$. In the case of an EE-contraction function - , then, it is possible to specify a very simple "withdrawal function" $\backslash$ over $K$ (the term is due to Makinson [8]) which is revision-equivalent over $K$ in the sense that $R(\backslash)=R(-)$. We make use of EE-cuts which have been helpful in simplifying some proofs of the last section. 
DEFINITION 7. If $\leq$ is an EE-relation on L, then the associated withdrawal function $\backslash=W(\leq)$ over $K$ is given by

$$
K \backslash A= \begin{cases}S_{A}=\{B: A<B\}, & \text { if } \forall A, \\ K, & \text { if } \vdash A .\end{cases}
$$

It is easy to see that has nearly all the properties of a contraction function:

LEMMA 6. Let $\leq$ be an EE-relation on $L$ (with respect to a knowledge set $K)$. Then $=W(\leq)$ satisfies $(C l)-(C 4)$ and $(C 6)-(C 8)$, but not $(\mathrm{C} 5)$.

Proof. For $\vdash A$ we have $K \backslash A=K$, and it is easy to verify (C1)(C8). So let $\forall A$. Then, by (E6) and (E2), $S_{A} \neq \varnothing$. We saw in Section 4 that non-empty cuts are knowledge sets, so $(\mathrm{C} 1)$ is satisfied. (C2) and (C3) follow from (E5). (C4) follows from the reflexivity of $\leq$ (which in turn is due to (E2)). (C6) follows from (E3). For (C7), assume that $C \in K \backslash A \cap K \backslash B$, i.e., $A<C$ and $B<C$. By (E3), $A \& B \leq A$, hence, by (E1), $A \& B<C$, so $C \in K \backslash A \& B$. (Note that even $K \backslash A \cup K \backslash B \subseteq K \backslash A \& B$ and that $K \backslash A \& B=K \backslash A$ or $K \backslash A \& B=K \backslash B$.) For (C8), assume that $A \notin K \backslash A \& B$ and $C \in K \backslash A \& B$, i.e., $A \leq A \& B$ and $A \& B<C$. Thus, by (E1), $A<C$, hence $C \in K \backslash A$.

We give a counterexample to (C5). Let $A, B \in \mathrm{L}$ such that $H^{H} A$ and $A \forall B$. We consider $K=\operatorname{Cn}(\{A, B\})$ and define $\leq$ on $\mathrm{L}$ by putting $T \leq C \leq \mathrm{T}$ for $C \in \mathrm{LT}, \perp \leq C \leq \perp$ for $C \notin K, \perp<C<\mathrm{T}$ for $C \in K-\mathrm{LT}, C \leq D$ and $D \leq C$ for $C, D \in K-\mathrm{LT}$, and then taking the transitive closure. It is easy to check that this generates an EE-relation on $\mathrm{L}$. We find that $\operatorname{Cn}((K \backslash A) \cup\{A\})=\operatorname{Cn}(\{C: A<C\} \cup$ $\{A\})=\operatorname{Cn}(\operatorname{LT} \cup\{A\})=\operatorname{Cn}(\{A\})$. Since by assumption $K-$ $\operatorname{Cn}(\{A, B\})$ and $A \forall B$, then, $K$ is not a subset of $\operatorname{Cn}((K \backslash A) \cup\{A\})$.

Given $=W(\leq)$ and $-=C(\leq)$ for some EE-relation $\leq$ on $\mathrm{L}$, it is clear that $K \backslash A \subseteq K-A$ : if $A<B$ then $B \in K$ by (E5) and $A<A \vee B$ by (E3) and (E1). But $K \backslash A$ gives up too many sentences 
(see the counterexample to (C5) just given). The violation of the recovery postulate $(\mathrm{C} 5)$ is a violation of the idea of minimal loss of information (see [8] for a thorough discussion of recovery). It is interesting to observe, however, that $\backslash$ is revision-equivalent to - :

LEMMA 7. Let $\leq$ be an EE-relation on L. Then $R(W(\leq))=$ $R(C(\leq))$.

Proof. We have to show that $\operatorname{Cn}\left(S_{A} \cup\{\neg A\}\right)=\operatorname{Cn}((K \cap\{B: A<$ $A \vee B\}) \cup\{\neg A\})$. We already know that $S_{A} \subseteq K \cap\{B: A<$ $A \vee B\}$ ), so it remains to show that RHS $\subseteq$ LHS. Suppose that $C \in \mathrm{RHS}$, i.e., that there are $B_{1}, \ldots, B_{n}$ such that $B_{i} \in K$ and $A<A \vee B_{i}$ for all $i=1, \ldots, n$ and $B_{1}, \ldots, B_{n}, \neg A \vdash C(\neg A$ may be redundant). Then obviously $A \vee B_{1}, \ldots, A \vee B_{n}, A \vee$ $\neg A \vdash A \vee C$. Since we may drop the premise $A \vee \neg A$ and since $A \vee B_{i} \in S_{A}$ for all $i=1, \ldots, n$, we find that $A \vee C \in \operatorname{Cn}\left(S_{A}\right)$, so $C \in \operatorname{Cn}\left(S_{A} \cup\{\neg A\}\right)$.

The upshot of Lemma 7 is that it is very easy to perform EE-revisions, i.e., revisions obtained from EE-contractions via the Levi identity. In order to go to $K+A$, just keep all those sentences of $K$ that are epistemically better entrenched than $\neg A$, add $A$ and take the deductive closure. There is no trouble with intricate comparisons of disjunctions as in Definition 2. This appears to be a very perspicuous and managable method of constructing revisions of knowledge bases.

\section{REMARKS ON ITERATED REVISIONS}

So far we have investigated transitions from some knowledge set $K$ to a revised knowledge set $K+A$. On closer inspection, however, it comes out that we started out from $K$ together with an EE-relation $\leq$ with respect to $K$ but ended up with a bare set of sentences. There is no guidance at all what to do if we were to revise $K+A$ in a second step. We need an EE-relation $\leq_{A}$ with respect to $K+A$ to accomplish iterated revisions. To put it differently, it is not knowledge sets that have to be revised but EE-relations. ${ }^{6}$ We can indeed get something like revisions of FF-relations in the Gärdenfors model, but they are not perfect. I believe the most suitable suggestion is this: 
DEFINITION 8. Let $\leq$ be an EE-relation on $\mathrm{L}$ (with respect to a knowledge of set $K$ ). Then the revision $\leq_{A}$ of $\leq$ is given by

$$
B \leq_{A} C \text { if and only if } A \rightarrow B \leq A \rightarrow C .
$$

To form an idea of how this definition works, we note the following properties of $\leq_{A}$ :

LEMMA 8. Let $\leq$ be an EE-relation on $L$ (with respect to knowledge set $K$ ).

(i) Then $\leq_{A}$ satisfies (E1)-(E4).

(ii) Let it $\neg A$. Then $\leq_{A}$ and $K+A$ satisfy (E5) if and only if $K+A=K \dot{+}^{*} A$ where $\dot{+}^{*}=R(C(\leq))$.

(iii) Let $\forall A$. Then $\leq_{A}$ does not satisfy (E6).

Proof. (i) It is straightforward to show that $\leq_{A}$ inherits (E1)-(E4) from the corresponding postulates for $\leq$.

(ii) Let $\leq_{A}$ and $K+A$ satisfy (E5). That is,

$$
C<{ }_{A} B \text { for some } C \text { if and only if } B \in K+A \text {. }
$$

By Definition 8, the LHS of $\left({ }^{*}\right)$ means that $A \rightarrow C<A \rightarrow B$ for some $C$ which, by (E3), is equivalent to $\neg A<A \rightarrow B$, or $A \rightarrow$ $B \in S_{\neg, A}$. We recall from Section 4 that the asumption $\vdash \rightarrow A$ implies that $S_{\neg A} \neq \varnothing$ is a theory. So $A \rightarrow B \in S_{\neg A}$ is equivalent to $B \in$ $\mathrm{Cn}\left(S_{\neg A} \cup\{A\}\right)$. Comparing this with the RHS of $\left({ }^{*}\right)$, we can transform the postulate (E5) for $\leq_{A}$ and $K+A$ into $K+A=$ $\operatorname{Cn}\left(S_{\neg A} \cup\{A\}\right)$. By Lemma 7, this means that $K+A=K \dot{+}^{*} A$ where $\dot{+}^{*}=R(C(\leq))$, and we are done.

(iii) By (E3), $A \rightarrow B \leq A \rightarrow A$ for all $B$, hence $B \leq_{A} A$ for all $B$, despite the assumption that $\Vdash A$. So $\leq_{A}$ violates (E6).

The only point that does not fit into the nice picture is mentioned in part (iii). On the one hand, I do not think that the violation of maximality is very annoying. (E6) is a limiting case postulate which could be dropped if some suitable technical changes were made at other places in the revision model. If there are contingent $A$ 's such that $A \leq \perp$ (the $A$ 's outside $K$ ), why shouldn't there be contingent $A$ 's such that $\mathrm{T} \leqslant A$ ? Candidates for sentences pretending to maximal 
epistemic entrenchment are 'Bachelors are unmarried', 'Penguins are birds', or ' $F=m \cdot a$ ' for an advocate of Newtonian mechanics.

On the other hand, the way how Definition 8 invalidates maximality is dissatisfying. Evidently, $A$ is a greatest element under $\leq_{A}$, by $\vdash A \rightarrow A$ and (E3). So $A$ is put on the same level as the theorems of the logic $\mathrm{Cn}$ in $K+A$. Worse, $A$ cannot lose this extraordinary status through later revisions, which is again due to Definition 8 . For instance, we cannot make plausible statements about $(K+A)+\neg A$. Because $A$ is extraordinary in $K+A$, the EE-revision $(K+A)+$ $\neg A$ is $\operatorname{Cn}\left(\left\{B: A<_{A} B\right\} \cup\{\neg A\}\right)=\operatorname{Cn}(\neg A)$, while intuitively we rather expect $(K+A)+\neg A=K+\neg A$ if $\forall \neg A$. Difficulties like this are, $I$ believe, not to be ascribed in some awkwardness of Definition 8 , but to the fundamental limitations of the purely relational EErevision model. Corresponding difficulties are known in probability theory, where standard conditionalization gives $P_{A}(A)=1$, and no later conditionalization of $P_{A}$ will push the probability of $A$ down any more. A well-known solution of this problem is to employ the Jeffrey conditionalization $P_{A, \alpha}=\alpha P_{A}+(1-\alpha) P_{\neg A}$ for some certainty factor $\alpha$ between 0 and 1 . It seems that in order to tackle the analogous problem for non-probabilistic revisions the purely relational structure of knowledge bases must be enriched to an ordinal one. This task is accomplished by Spohn's $[9,10]$ theory of ordinal conditional functions.?

\section{NOTES}

1 Isaac Levi made me realize that there are contexts where the recovery postulate (C5) is problematic. In such contexts the constructions to be discussed do not seem appropriate. Compare [8].

${ }^{2}$ In [3] and [4], $M(K)$ is defined as $\bigcup\{M(K, A): A \in K$ and $\forall A\}$, and in [2] as $\bigcup\{M(K, A): A \in K\}$.

${ }^{3}$ In many contexts (P0) is not really essential. By defining $K-A$ as $\bigcup\left\{\cap\left\{M^{\prime} \in M(K, A)\right.\right.$ : $\left.\left.M^{\prime}>M\right\}: M \in M(K, A)\right\}$ one could dispense with (P0) and nevertheless get most of the things desired. I shall keep (P0), though, partly for the sake of simplicity and partly because I need it in the proof of my central Theorem 3.

4 (E4) is not needed for the proof of this result. It is also remarkable that (P0) is not even mentioned in Gärdenfors [3].

5 The concept of an EE-cut is inspirad by, but not identical with the concept of a cut in Grove [6], p. 165. 
${ }^{6}$ The concept of a knowledge set is, in a sense, superfiuous. One could take EErelations on $L$ as primitive and define associated knowledge sets $K \neq L$ by $K=S_{\perp}$. Similarly, taking P-relations $\leqslant$ on $2^{\mathrm{L}}$ or contraction functions - from $\mathrm{L}$ to $2^{\mathrm{L}}$ as primitive, one could get knowledge sets $K \neq \mathrm{L}$ by putting $K=\bigcap\left\{M \in 2^{\mathrm{L}}: L \leqslant M\right\}$ and $K=\dot{-(1)}$ respoctively.

'I want to thank Isaac Levi and David Makinson for a number of helpful comments.

\section{REFERENCES}

[1] Alchourrón, Carlos E. and David Makinson, 'On the Logic of Theory Change: Contraction Functions and Their Associated Revision Functions', Theoria 48 (1982) 14-37.

[2] Alchourrón Carlos E., Peter Gärdenfors, and David Makinson, 'On the Logic of Theory Change: Partial Meet Contraction and Revision Functions', Journal of Symbolic: Logic 50 (1985), 510-530.

[3] Gärdenfors, Peter, 'Epistemic Importance and the Logic of Theory Change', in Georg J. W. Dorn and Paul Weingartner (eds.), Foundations of Logic and Linguistics - Problems and Their Solutions, Plenum Press, New York, London, 1984, 345-367.

[4] Gärdenfors, Peter, Knowledge in Flux: Modeling the Dynamics of Epistemic States, Cambridge/Mass., London, Bradford Books, 1988.

[S] Gärdenfors, Peter and David Makinson, 'Revisions of Knowledge Systems Using Epistemic Entrenchment', in Moshe Y. Vardi (ed.), Theoretical Aspects of Reasoning about Knowledge, Morgan Kaufmann, Los Altos/California, 1988, 83-95.

[6] Grove, Adam, 'Two Modellings for Theory Change', Journal of Philosophical Logic 17 (1988), 157-170.

[7] Makinson, David, 'How to Give It Up: A Survey of Some Formal Aspects of the Logic of Theory Change', Synthese 62 (1985), 347-363. (Errata in Synthese 68 (1986), 185-186.)

[8] Makinson, David, 'On the Status of the Postulate of Recovery in the Logic of Theory Change', Journal of Philosophical Logic 16 (1987), 383-394.

[9] Spohn, Wolfgang, 'Ordinal Conditional Functions', in William L. Harper and Brian Skyrms (eds.), Cousation in Decision, Belief Change, and Statistics, vol. II, $1988,105-134$.

[10] Spohn, Wolfgang, 'A General Non-Probabilistic Theory of Inductive Reasoning', Proceedings of the 4th Workshop on Uncertainty in Artificial Intelligence. University of Minnesota, Minneapolis, 1988, 315-322.

University of Stuttgart,

Institute of Computational Linguistics

Formal Logic and Philosophy of Language,

Keplerstr. 17,

D-7000 Stuttgart 1,

Germany 